\section{Medical Principles and Practice}

Med Princ Pract 2012;21:383-388

DOI: $\underline{10.1159 / 000335277}$
Received: March 28, 2011

Accepted: November 20, 2011

Published online: January 13, 2012

\title{
A Survey of Psychiatrists' Expectations of Clinical Pharmacists in Riyadh, Saudi Arabia
}

\author{
S. Al-Aqeel ${ }^{\mathrm{a}} \quad$ J. Al-Sabhan ${ }^{\mathrm{a}}$ K. Al-Jumah ${ }^{\mathrm{b}}$

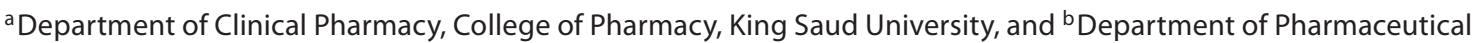 \\ Care, Al-Amal Complex for Mental Health, Riyadh, Saudi Arabia
}

\section{Key Words}

Pharmacists - Psychiatry - Questionnaires - Attitude of health personnel $\cdot$ Interprofessional relations $\cdot$ Saudi Arabia

\begin{abstract}
Objectives: To measure psychiatrists' experience and their expectations of the role of clinical pharmacists in Riyadh, Saudi Arabia. Subjects and Methods: The psychiatrists' experience and their expectations were collected using a 4-part self-completed questionnaire: (a) demographic information, (b) psychiatrists' current expectations of the roles of pharmacists, (c) psychiatrists' actual experience with pharmacists, and (d) psychiatrists' future expectations of pharmacists' roles. Each part included a set of statements for which respondents were asked to indicate their level of agreement using a 5-point Likert scale. Results: Of the 102 questionnaires delivered to the Department of Psychiatry at 7 hospitals, 77 were returned, representing a response rate of $75 \% .59$ (77\%) psychiatrists expected pharmacists to take personal responsibility for resolving any drug-related problems; 62 (81\%) expected them to maintain a complete medication profile, and 58 (75\%) expected them to educate patients about the safety and appropriate use of their medication. From their current experience with pharmacists, respondents indicated that pharmacists routinely counsel
\end{abstract}

patients $(33,43 \%)$, inform them about the most cost-effective alternative $(37,48 \%)$, and discuss with them the therapeutic value of the drugs (37, 48\%). 49 (63\%) of respondents agreed that in the future, pharmacists should routinely adjust the drug therapy for patients under protocol approved by the physician, and 51 (66\%) felt that pharmacists should share legal responsibility for clinical outcomes. Conclusion: Psychiatrists in Riyadh had great expectations of clinical pharmacists to take personal responsibility for resolving drug-related problems they discovered and to assist in designing drug therapy treatment plans. The psychiatrists' current experience with pharmacists did not, in some cases, meet their expectations.

Copyright $\odot 2012$ S. Karger AG, Basel

\section{Introduction}

Psychiatry in Saudi Arabia has changed significantly since the review published in 1983 by Dubovsky [1] where he referred to Shehar, the only mental hospital serving the population of Saudi Arabia back then. Nowadays, it is estimated that there are 20 psychiatric hospitals in addition to 44 psychiatric clinics in general hospitals [2]. There are no published figures on the prevalence of mental illness in Saudi Arabia; however, recent data indicate that there

\section{KARGER}

Fax +4161306 1234

E-Mail karger@karger.ch

www.karger.com (c) 2012 S. Karger AG, Basel

$1011-7571 / 12 / 0214-0383 \$ 38.00 / 0$

Accessible online at:

www.karger.com/mpp
Sinaa Al-Aqeel

Department of Clinical Pharmacy, College of Pharmacy, King Saud University PO Box 376316

Riyadh 11335 (Saudi Arabia)

Tel. +9661506108592, E-Mail salageel@ksu.edu.sa 
are approximately 439,762 outpatients and 17,811 inpatients with behavioral and mental disorders at the Ministry of Health $(\mathrm{MoH})$ hospitals alone [3].

Recently, experts and influential organizations such as the World Health Organization have supported the increased role of pharmacists in the care of mentally ill patients [4]. A systematic review of the literature from 1972 to 2003 on pharmacists' impact in mental illness demonstrated improvements in outcomes, prescribing practices, patient satisfaction, and resource use [5]. Another systematic review showed that pharmacist-delivered community-based services contribute to optimizing the use of medications for mental illness [6]. Furthermore, a recent systematic review found a significant benefit from pharmacist interventions in the improvement of adherence to antidepressant pharmacological treatment [7].

Previous research has indicated that when pharmacists and physicians jointly specify roles and responsibilities, the relationship is more likely to become collaborative $[8,9]$. Understanding physicians' expectations of pharmacists, therefore, should improve the collaboration between pharmacists and physicians. Several authors have reported physicians' expectations of pharmacists in community [10-12] and hospital [12-16] settings, but not in the Saudi context.

In view of the limited information on the subject in Saudi Arabia, this research aims to measure physicians' experience with and expectations of clinical pharmacists in Riyadh, Saudi Arabia, with particular interest in psychiatrists. The concept of clinical pharmacy is not new to Saudi Arabia. The teaching of clinically oriented courses to pharmacy students in Saudi Arabia started in the 1980s by the first and only pharmacy college at that time. The same college initiated a clinical pharmacy master degree in the 1990s. Recent estimates indicate that there are approximately 4,400 pharmacists working in governmental and private sectors excluding those working in community pharmacies [3]. However, not all these pharmacists work as clinical pharmacists. To work as clinical pharmacists in Saudi Arabia, you should have a doctor of pharmacy (PharmD) degree, a master in clinical pharmacy, or completed a pharmacy practice residency program.

\section{Subjects and Methods}

\section{Sample}

The target population of the study was psychiatrists working in government hospitals in Riyadh city, the capital of Saudi Arabia. There are 7 government hospitals with psychiatry services in
Riyadh: 1 specialized psychiatric hospital and 6 psychiatric clinics. Published data indicate that the number of psychiatrists working in Riyadh for $\mathrm{MoH}$ hospitals was 68 . The number of psychiatrists working in other governmental non-MoH hospitals was 100; however, this number included neurologists [3]. Therefore, to determine the exact number of psychiatrists, we contacted the Departments of Psychiatry in each of the target hospitals.

\section{Data Collection}

The data were collected using a self-completed questionnaire in English as English is the main language of communication in Saudi hospitals. The questionnaire was divided into 4 sections. Section 1 collected demographic information including the respondents' type of training (Saudi program, Arabic program, American program, or others) and experience. Sections 2, 3, and 4 included a set of statements to measure respondents' current expectations, actual experience, and future expectations of pharmacists, respectively. The respondents were asked to indicate their level of agreement with each statement using a 5-point Likert scale from strongly disagree to strongly agree. Statements used were developed by Smith et al. [12] and previously used by other investigators [14-16]. The questionnaire was pretested among 5 nonpsychiatrist physicians, for readability and understanding.

\section{Distribution of the Questionnaires}

102 questionnaires were hand delivered by one of the authors (J.S.) to the secretary of the Department of Psychiatry in the target hospitals to be passed on to the psychiatrists. The completed questionnaires were collected by hand by the same author. The data collection period was from January to March 2008. We did not use follow-up phone calls or reminder letters to enhance response rate. Formal ethical permission was not required for this study. The covering letter stressed anonymity and confidentiality. The consent to participate was implied by the act of completing and returning the questionnaire.

\section{Statistical Analysis}

The data were analyzed using descriptive statistics: frequency, percentage, mean, and standard deviation. Data were entered and analyzed using SPSS version 15 (SPSS Inc., Chicago. Ill., USA).

\section{Results}

Of the 102 questionnaires, 77 were returned representing a response rate of $75 \%$. The majority of respondents were specialists trained in an Arabic country working for the $\mathrm{MoH}$ hospitals and with an experience of more than 15 years (table 1). 53 (69\%) respondents stated that their hospitals provide clinical services.

\section{Current Expectations}

The results of psychiatrists' expectations of pharmacists are listed in table 2. $56(73 \%)$ respondents expect pharmacists to be drug therapy experts and 63 (82\%) expect them to know the specific indication(s) for the pre- 
scribed medications. 62 (81\%) respondents expect pharmacists to maintain a complete medication profile, 57 (74\%) to help in designing drug therapy treatment plans, 67 (87\%) to perform therapeutic drug monitoring for selected drugs, 59 (77\%) to take personal responsibility for resolving any drug-related problems, and 67 (87\%) to be available during rounds.

\section{Current Experience}

Respondents' current experiences with pharmacists are given in table 3. 51 (66\%) of the respondents agree that pharmacists are reliable sources of clinical drug information, 50 (65\%) think that they should inform doctors if patients have experienced some problem with their medications, and 48 (62\%) of the pharmacists are willing to take personal responsibility for resolving drugrelated problems they have discovered. 33 (43\%) also agree that pharmacists routinely counsel patients and 37 (48\%) that they inform doctors of the most cost-effective alternative.

\section{Future Expectations}

The results of future expectations are shown in table 4 . $49(63 \%)$ of the respondents agree that in the future, pharmacists should routinely adjust the drug therapy for patients under protocol approved by the physician. 51 (66\%) respondents agree that pharmacists should share legal responsibility for clinical outcomes.

\section{Discussion}

This study examined the psychiatrists' experience with and expectations of clinical pharmacists in Riyadh, Saudi Arabia. The results from this survey do not differ greatly from the surveys of other physicians' expectation of pharmacists to be knowledgeable drug therapy experts [12-16]. 75\% of respondents expect clinical pharmacists to educate patients about the safe and appropriate use of their medication and this reflects expectations reported elsewhere [12-17]. Interestingly, a Finish survey on physicians' opinions on community pharmacists' involvement in counseling patients on the use of psychotropic medication found that 75 and $60 \%$ of the physicians felt that community pharmacists should provide information about the adverse effects of benzodiazepines and neuroleptics, respectively. However, only 43 and $35 \%$ of the physicians felt that community pharmacists should provide information about the purpose of the medication of benzodiazepines and neuroleptics, respectively [18].
Table 1. Demographic characteristics of respondents

\begin{tabular}{lrr}
\hline Characteristics & $\mathrm{n}$ & $\%$ \\
\hline Type of hospital & & \\
$\quad$ MOH & 45 & 59 \\
$\quad$ Government non-MOH & 32 & 41 \\
Professional position & & \\
$\quad$ Specialist & 39 & 50 \\
$\quad$ Consultant & 17 & 22 \\
$\quad$ Residence & 11 & 14 \\
$\quad$ Registrar & 9 & 11 \\
Type of training & & \\
Arabic program & 46 & 60 \\
Saudi program & 11 & 14 \\
American program & 4 & 5 \\
Others & 14 & 18 \\
Years of experience & & \\
$\quad<5$ & 7 & 9 \\
$5-10$ & 29 & 38 \\
10-15 & 16 & 21 \\
$>15$ & 24 & 31 \\
\hline
\end{tabular}

Psychiatrists appeared receptive to pharmacists' direct patient care roles such as help in designing drug therapy treatment plans, monitor patients' response to drug therapy, and take personal responsibility for resolving drugrelated problems they discover. In previous surveys, physicians were less receptive with only $33-61 \%$ who expect pharmacists to perform these particular services $[12,14-$ 16]. Several explanations for the more positive expectations reported in our survey are possible. One probable reason is that other surveys investigated pharmacists in general, not necessarily clinically trained pharmacists.

Another probable explanation is that our study focused on psychiatry, an area which is characterized by multiple drug therapy, use of drugs with a broad range of side effects, and likelihood of drug-drug interactions. In a survey of a similarly complex care setting, Fairbanks et al. [13] surveyed 75 physicians and nurses to assess their acceptance of services provided by residency-trained clinical pharmacists based in the emergency department. They found that over $80 \%$ of respondents felt that pharmacists should help with the selection of the appropriate antibiotic, advice regarding which is the most appropriate medication, and make medication decisions on the basis of medication pricing or efficacy. The findings from a qualitative Australian study similarly suggest that mental health care providers welcome the participation of pharmacists in mental health teams' meetings and medication review and valued the provision of information 
Table 2. Psychiatrists' current expectations of pharmacists

Survey item Strongly Agree Neutral Disagree Strongly agree

I expect clinical pharmacists to take personal responsibility for resolving any drug-related problems they discover

I expect clinical pharmacists to maintain a complete medication profile on my patients (i.e. medication history, allergy history)

I expect clinical pharmacists to advise me about more cost-effective alternatives to the drugs I prescribe

I expect clinical pharmacists to be knowledgeable drug therapy experts

I expect clinical pharmacists to assist me in designing drug therapy treatment plans for my patients

I expect clinical pharmacists to educate my patients about the safe and appropriate use of their medication

I expect clinical pharmacists to monitor my patients' response to drug therapy

I expect clinical pharmacists to know the specific indication of each drug I prescribe, even when drugs have more than one approved indication

I expect clinical pharmacists to be available to me for consultation during rounds

I expect clinical pharmacists to perform therapeutic drug monitoring for my patients for selected drugs

$\begin{array}{lllll}20(26) & 39(51) & - & 18(23) & - \\ 15(20) & 47(61) & 3(4) & 12(15) & - \\ 19(28) & 37(48) & 6(7) & 10(13) & 5(6) \\ 17(22) & 39(51) & 9(12) & 12(16) & - \\ 10(13) & 47(61) & 10(13) & 5(7) & 5(7) \\ 24(31) & 34(44) & 7(9) & 9(12) & - \\ 4(5) & 37(48) & 16(20) & 19(25) & - \\ 17(22) & 46(60) & 6(8) & 7(9) & - \\ 16(21) & 51(66) & 3(6) & 5(7) & - \\ 12(16) & 55(71) & 2(3) & 7(9) & -\end{array}$

Figures are numbers with percentages in parentheses. The total percentage does not add up to $100 \%$ because of missing responses.

Table 3. Psychiatrists' current experience with pharmacists

Survey item

Strongly Agree Neutral Disagree Strongly

agree

In my experience, clinical pharmacists are a reliable source of general drug information

In my experience, clinical pharmacists are a reliable source of clinical drug information

Clinical pharmacists routinely counsel my patients regarding the safe and appropriate use of their medications

Clinical pharmacists routinely inform me if they discover clinical problems with my prescriptions

Clinical pharmacists routinely inform me about less expensive alternatives to the drugs I prescribe

Clinical pharmacists routinely inform me about more cost-effective alternatives to the drugs I prescribe

Clinical pharmacists frequently ask me to clarify for them the drug therapy objectives I have in mind for my patients

Clinical pharmacists frequently let me know that my patients have experienced some problem with their medication

In my experience, clinical pharmacists appear willing to take personal responsibility for resolving drug-related problems they discover

$\begin{array}{lllll}54(70) & - & 16(21) & 5(7) & - \\ 3(4) & 48(62) & 22(29) & - & - \\ 3(4) & 30(39) & 32(42) & 7(9) & 3(4) \\ 2(3) & 46(60) & 12(16) & 5(7) & 8(10) \\ 4(5) & 39(51) & 14(18) & 10(13) & 8(10) \\ 37(48) & - & 17(22) & 13(17) & 8(10) \\ 3(4) & 34(44) & 13(17) & 11(14) & 13(17) \\ 1(1) & 49(64) & - & 17(22) & 8(10) \\ 1(1) & 47(61) & 15(20) & 5(6) & 7(9)\end{array}$

Figures are numbers with percentages in parentheses. The total percentage does not add up to $100 \%$ because of missing responses. 
Table 4. Psychiatrists' future expectations of clinical pharmacists

\begin{tabular}{|c|c|c|c|c|c|}
\hline Survey item & $\begin{array}{l}\text { Strongly } \\
\text { agree }\end{array}$ & Agree & Neutral & Disagree & $\begin{array}{l}\text { Strongly } \\
\text { disagree }\end{array}$ \\
\hline $\begin{array}{l}\text { In the future, I would like clinical pharmacists to routinely adjust the drug } \\
\text { therapy for some of my patients using protocols that I have approved }\end{array}$ & $10(13)$ & $39(50)$ & $13(17)$ & - & $5(7)$ \\
\hline $\begin{array}{l}\text { In the future, I would like clinical pharmacists to be on-call to my patients } \\
\text { for questions about their medications }\end{array}$ & $12(17)$ & $24(31)$ & $13(17)$ & $19(25)$ & - \\
\hline $\begin{array}{l}\text { Clinical pharmacists in the future should monitor and document my patients' } \\
\text { response to drug therapy }\end{array}$ & $14(18)$ & $43(56)$ & $4(5)$ & $5(7)$ & $1(1)$ \\
\hline $\begin{array}{l}\text { Clinical pharmacist in the future should bear a greater financial responsibility } \\
\text { for patients' drug therapy outcomes }\end{array}$ & $7(9)$ & $28(36)$ & $21(27)$ & $8(10)$ & $4(5)$ \\
\hline $\begin{array}{l}\text { Clinical pharmacists in the future should bear a greater share of legal } \\
\text { responsibility for the outcomes of drug therapy }\end{array}$ & $20(26)$ & $31(40)$ & $10(13)$ & $5(7)$ & $1(1)$ \\
\hline
\end{tabular}

Figures are numbers with percentages in parentheses. The total percentage does not add up to $100 \%$ because of missing responses.

about medications by pharmacists to clients, caregivers, and families [19].

As with previous surveys [12, 14-16], this study found that pharmacists appear not to be measuring up to psychiatrists' expectations in many domains that included uncertainty of clinical pharmacists routinely counseling patients regarding the safe and appropriate use of their medication, and communicating patients' experiences with their medication. One possible explanation of the mismatch between expectation and current experience is the shortage of clinical pharmacists, as only $69 \%$ respondents stated that their hospitals provide clinical services. Hopefully, shortage of pharmacists will not be a problem in the future as the number of pharmacy colleges in Saudi Arabia has increased from 3 to 20 over the last 6 years and the majority of these colleges offer a PharmD degree. Another probable explanation could be pharmacists' perceived attitudes and barriers to providing pharmaceutical care to mental health patients. Pharmacists have positive attitudes toward both mental illness and the providing of pharmaceutical care to mentally ill patients [20,21]; however, lack of adequate education in mental health issues have been reported as an important barrier to the provision of pharmaceutical care [22]. Surveys of the psychiatric pharmacy curriculum in the USA and Europe showed that the psychiatryrelated topics are taught as part of a therapeutics-based course and a fewer number of colleges offer internship in psychiatry $[23,24]$. This is somewhat similar to the current practice in Saudi Arabia. The teaching of psychiatry-related topics should ensure the pharmacy graduates have the knowledge, skills, and capacity to deliver a wider range of patient-oriented services. In the USA, a residency requirement has been proposed as a prerequisite for all pharmacists providing direct patient care [25], although others argue that all PharmD graduates should be able to provide a certain level of direct patient care, without completing a residency [26]. There is, however, agreement that residency programs should be offered for complex levels of direct patient care in all practice settings [26]. The Saudi Commission for Health Specialties offers 1 advanced practice specialty [27] while the US Board of Pharmaceutical Specialties recognizes 6 advanced practice specialties: psychiatry, oncology, nutritional support, nuclear therapy, ambulatory care, and pharmacotherapy [28]. Thus, we suggest that the Saudi Commission for Health Specialties introduce more specialty board certifications for complex levels including psychiatry to meet the workforce needs for specialtytrained pharmacists.

The study has several limitations: the use of a selfcompleted questionnaire means that the results depend on the respondents' interpretation of the questionnaire items; the possibility of a social response bias that could tempt the respondents to give a socially desirable response rather than what they think or believe. Assurance of confidentiality may have helped to reduce the social desirability bias. Second, the external validity of the findings may be questioned as we do not know of the differences in demographic characteristics between responders and nonresponders. The response to this study was voluntary; it is therefore possible that only psychiatrists 
who were interested in pharmacists' services likely filled in the questionnaire. Nevertheless, the responding psychiatrists represent different hospitals and different professional positions with a variety of experience and training. Third, our data came from a representative sample of psychiatrists of one city of Saudi Arabia, limiting generalizability to the entire country.

\section{Conclusion}

Our survey suggests that psychiatrists are receptive to many elements of patient-centered pharmaceutical services. However, respondents' current experience with pharmacists does not, in some cases, meet their expectations. Pharmacists need to close the gap between services they currently provide and psychiatrists' expectations. The pharmacy education and training program should be tailored to provide pharmacists with the necessary skills required to provide these services.

\section{References}

1 Dubovsky SL: Psychiatry in Saudi Arabia. Am J Psychiatry 1983;140:1455-1459.

-2 Al-Habeeb AA, Qureshi NA: Mental and social health atlas I in Saudi Arabia: 2007-08. East Mediterr Health J 2010;16:570-577.

3 The Ministry of Health Statistics Book. Ministry of Health, 2009. http://www.moh.gov. sa/en/Ministry/Statistics/book/Pages/default.aspx (accessed July 7, 2011).

4 World Health Organization: Improving Access and Use of Psychotropic Medicines. Geneva, World Health Organization, 2004. http://whqlibdoc.who.int/publications/2004/9241546417.pdf (accessed July 7, 2011).

5 Finley PR, Crismon ML, Rush AJ: Evaluating the impact of pharmacists in mental health: a systematic review. Pharmacotherapy 2003; 23:1634-1644.

6 Bell S, McLachlan AJ, Aslani P, Whitehead P, Chen TF: Community pharmacy services to optimise the use of medications for mental illness: a systematic review. Aust New Zealand Health Policy 2005;2:29.

-7 Rubio-Valera M, Serrano-Blanco A, Magdalena-Belio J, Fernandez A, Garcia-Campayo J, Pujol MM, del Hoyo YL: Effectiveness of pharmacist care in the improvement of adherence to antidepressants: a systematic review and meta-analysis. Ann Pharmacother 2011;45:39-48.

-8 Doucette WR, Nevins J, McDonough RP: Factors affecting collaborative care between pharmacists and physicians. Res Social Adm Pharm 2005;1:565-578.

-9 Zillich AJ, McDonough RP, Carter BL, Doucette WR: Influential characteristics of physician/pharmacist collaborative relationships. Ann Pharmacother 2004;38:764-770.

10 Bryant LJ, Coster G, Gamble GD, McCormick RN: General practitioners' and pharmacists' perceptions of the role of community pharmacists in delivering clinical services. Res Social Adm Pharm 2009;5:347362
11 Alkhateeb FM, Unni E, Latif D, Shawaqfeh MS, Al-Rousan RM: Physician attitudes toward collaborative agreements with pharmacists and their expectations of community pharmacists' responsibilities in West Virginia. J Am Pharm Assoc 2009;49:797-800.

12 Smith WE, Ray MD, Shannon DM: Physicians' expectations of pharmacists. Am J Health Syst Pharm 2002;59:50-57.

13 Fairbanks RJ, Hildebrand JM, Kolstee KE, Schneider SM, Shah MN: Medical and nursing staff highly value clinical pharmacists in the emergency department. Emerg Med J 2007;24:716-718

14 Awad A, Matowe L, Capps P: Medical doctors' perceptions and expectations of the role of hospital pharmacists in Sudan. Pharm World Sci 2007;29:557-764.

15 Matowe L, Abahussain EA, Al-Saffar N, Bihzad SM, Al-Foraih A, Al-Kandery AA: Physicians' perceptions and expectations of pharmacists' professional duties in government hospitals in Kuwait. Med Princ Pract 2006; 15: 185-189.

16 Tahaineh LM, Wazaify M, Albsoul-Younes A, Khader Y, Zaidan M: Perceptions, experiences, and expectations of physicians in hospital settings in Jordan regarding the role of the pharmacist. Res Social Adm Pharm 2009;5:63-70.

17 Farrell B, Pottie K, Woodend K, Yao V, Dolovich L, Kennie N, Sellors C: Shifts in expectations: evaluating physicians' perceptions as pharmacists become integrated into family practice. J Interprof Care 2010;24:80-89.

18 Tanskanen P, Airaksinen M, Tanskanen A, Enlund $\mathrm{H}$ : Counselling patients on psychotropic medication: physicians' opinions on the role of community pharmacists. Pharm World Sci 2000;22:59-61.

19 Bell JS, Rosen A, Aslani P, Whitehead P, Chen TF: Developing the role of pharmacists as members of community mental health teams: perspectives of pharmacists and mental health professionals. Res Social Adm Pharm 2007;3:392-409.
20 Cates ME, Burton AR, Woolley TW: Attitudes of pharmacists toward mental illness and providing pharmaceutical care to the mentally ill. Ann Pharmacother 2005;39: 1450-1455.

-21 Phokeo V, Sproule B, Raman-Wilms L: Community pharmacists' attitudes toward and professional interactions with users of psychiatric medication. Psychiatr Serv 2004;55: 1434-1436.

22 Scheerder G, De Coster I, Van Audenhove C: Pharmacists' role in depression care: a survey of attitudes, current practices, and barriers. Psychiatr Serv 2008;59:1155-1160.

23 Cates ME, Monk-Tutor MR, Drummond SO Mental health and psychiatric pharmacy instruction in US colleges and schools of pharmacy. Am J Pharm Educ 2007;71:1-5.

24 Koski I, Heikkila A, Bell JS: Mental health pharmacy education at 16 European universities. Am J Pharm Educ 2009;73:139.

25 Murphy JE, Nappi JM, Bosso JA, Saseen JJ, Hemstreet BA, Halloran MA, Spinler SA, Welty TE, Dobesh PP, Chan LN, Garvin CG, Grunwald PE, Kamper CA, Sanoski CA, Witkowski PL: American College of Clinical Pharmacy's vision of the future: postgraduate pharmacy residency training as a prerequisite for direct patient care practice. Pharmacotherapy 2006;26:722-733.

26 Nahata MC: Residency requirement for pharmacists providing direct patient care. Ann Pharmacother 2010;44:1822-1825.

27 The Saudi Commission for Health Specialities. http://english.scfhs.org.sa/index.php? option=com_content\&view=category\&layout=blog\&id $=461 \&$ Itemid $=5000 \quad$ (accessed July 7, 2011).

28 The Board of Pharmacy Specialties. http:// www.bpsweb.org/about/councils.cfm (accessed July 7, 2011). 